\title{
Deletion of retinoic acid-related orphan receptor gamma reduces body weight and hepatic lipids in mice by modulating the expression of lipid metabolism genes
}

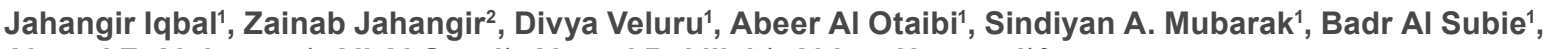 \\ Ahmad F. Alghanem¹, Ali Al Qarni', Ahmed Bakillah'1, Abbas Hawwari ${ }^{113}$ \\ 1King Abdullah International Medical Research Center-Eastern Region, King Saud Bin Abdulaziz University for Health Sciences, \\ Ministry of National Guard-Health Affairs, Al Ahsa 31982, Saudi Arabia. \\ 2JP Stevens High School, North Edison, NJ 08820, USA. \\ ${ }^{3}$ Department of Genetics, King Faisal Specialist Hospital and Research Center, Riyadh 11211, Saudi Arabia.
}

Correspondence to: Dr. Jahangir lqbal and Dr. Abbas Hawwari, King Abdullah International Medical Research Center, King Saud bin Abdulaziz University for Health Sciences, King Abdulaziz Medical City Hospital, Ministry of National Guard Health Affairs, Mail Code 520, Al Ahsa 31982, Saudi Arabia.E-mail: iqbalja@ngha.med.sa; hawwariab@ngha.med.sa

How to cite this article: Iqbal J, Jahangir Z, Veluru D, Otaibi AA, Mubarak SA, Subie BA, Alghanem AF, Qarni AA, Bakillah A, Hawwari A. Deletion of retinoic acid-related orphan receptor gamma reduces body weight and hepatic lipids in mice by modulating the expression of lipid metabolism genes. Vesse/ Plus 2019;3:40. http://dx.doi.org/10.20517/2574-1209.2019.28

\section{Received: 5 Dec 2019 First Decision: 9 Dec 2019 Revised: 9 Dec 2019 Accepted: 16 Dec 2019 Published: 26 Dec 2019}

Science Editor: Mario F. L. Gaudino Copy Editor: Jing-Wen Zhang Production Editor: Tian Zhang

\begin{abstract}
Aim: Retinoic acid-related orphan receptor $\gamma(R O R \gamma)$ functions as a ligand-dependent transcription factor and its loss has been shown to affect the circadian expression of lipid metabolism genes. However, its effect on body weight gain and hepatic lipids is not well understood. In this study, we investigated the impact of Rory gene deletion on changes in body weight and hepatic lipids.
\end{abstract}

Methods: Body weight and lipids were analyzed in the plasma and liver. Expression of lipid metabolism genes in the liver was evaluated in wild type and Rory knockout mice.

Results: We show that deletion of ROR $\gamma$ results in reduced body weight and fewer lipids in the liver. Analysis of gene expression showed that deletion of Rory resulted in an overall lower expression of genes and transcription factors involved in lipid biosynthesis. We observed a decrease in the gene expression of cholesterol biosynthesis, efflux, and esterification but an increase in bile acid synthesis. There was a decrease in fatty acid and triglycerides biosynthesis genes and an increase in the fatty acid uptake genes. The decrease in the expression of lipid biosynthesis genes was accompanied by the decrease in the sterol response element binding protein (Srebp) genes. We observed an increase in

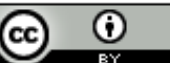

(C) The Author(s) 2019. Open Access This article is licensed under a Creative Commons Attribution 4.0 International License (https://creativecommons.org/licenses/by/4.0/), which permits unrestricted use sharing, adaptation, distribution and reproduction in any medium or format, for any purpose, even commercially, as long as you give appropriate credit to the original author(s) and the source, provide a link to the Creative Commons license, and indicate if changes were made. 
the expression of peroxisome proliferator-activated receptor alpha (Ppara) and a decrease in the expression of acetylCoA carboxylase 2 (Acc2) genes.

Conclusion: Our data suggest that RORy regulates body weight and lipid metabolism genes and its modulation may be beneficial for the management of obesity and related lipid metabolic disorders.

Keywords: Lipid metabolism, triglycerides, retinoic acid-related orphan receptor $\gamma$, peroxisome proliferator-activated receptors, sterol response element binding proteins

\section{INTRODUCTION}

Obesity is a major risk factor that leads to the development of other metabolic disorders such as hyperlipidemia and hepatic steatosis. Ectopic accumulation of fat in the liver causes insulin resistance by activating various cellular stress and inflammatory signaling pathways ${ }^{[1]}$. The liver plays an important role in lipid metabolism that involves several key proteins and transcription factors to maintain lipid homeostasis $^{[2]}$. Various cellular regulators that include several transcription factors control biosynthesis, oxidation, uptake, and secretion of lipids in the liver ${ }^{[3]}$. Peroxisome proliferator-activated receptors (PPARs), which serve as hepatic lipid sensors, have been demonstrated to play a critical role in lipid metabolism by controlling the enzymes through various signaling events ${ }^{[4,5]}$. Peroxisome proliferatoractivated receptor $\alpha(\mathrm{PPAR} \alpha)$ is highly expressed in the liver and is primarily involved in the regulation of enzymes crucial for fatty acid oxidation. It also plays a role in regulating genes that control fatty acid elongation, desaturation and transport ${ }^{[6]}$. On the other hand, peroxisome proliferator-activated receptor $\gamma(\operatorname{PPAR} \gamma)$, which is expressed at low levels in the liver ${ }^{[7]}$, controls the upregulation of a subset of the lipogenic genes that are involved in lipid synthesis and accumulation ${ }^{[8]}$. Besides PPARs, the sterol response element binding proteins (SREBPs) that act as nutrient sensing transcription factors are also involved in the transcriptional control of the lipogenic gene expression ${ }^{[9]}$. Sterol regulatory-element binding protein $1 \mathrm{c}$ (SREBP-1C) is a transcription factor that primarily upregulates the transcription of genes involved in fatty acid synthesis. In contrast, sterol regulatory-element binding protein 2 (SREBP-2) controls the activation of genes responsible mainly for cholesterol synthesis and uptake, as opposed to fatty acid synthesis. However, sterol regulatory-element binding protein 1a (SREBP-1a) leads to the activation of genes involved in both pathways $^{[10,11]}$.

The transcriptional networks of orphan nuclear receptors govern the hepatic metabolic pathways ${ }^{[12]}$. The retinoic acid-related orphan receptor $\gamma(\operatorname{ROR} \gamma)$, a member of the ROR subfamily of nuclear receptors, has been implicated in the control of a variety of physiological processes ${ }^{[13]}$. ROR $\gamma$ binds to specific RORresponsive elements at its genomic targets to constitutively activate the gene expression in the absence of a ligand ${ }^{[14]}$. Global ROR $\gamma$-deficient mice are born healthy and fertile but die within the first four months after birth due to high incidence of T-cell lymphomas in the thymus ${ }^{[15]}$. The physiological functions of ROR $\gamma$, expressed in various peripheral tissues ${ }^{[16]}$, are still poorly understood. ROR $\gamma$ has been shown to regulate the circadian expression of glucose and lipid metabolism genes ${ }^{[17,18]}$. Pharmacological inhibition of ROR $\gamma$ has been shown to reduce food intake and body weight gain ${ }^{[19]}$. However, effect of Ror $\gamma$ gene deletion on body weight and hepatic lipids is not well studied. Therefore, in this study, we investigated the effect of Rory gene deletion on body weight and hepatic lipids using a knockout $(\mathrm{KO})$ mouse model. We also studied the expression of downstream lipid metabolism genes that are regulated directly or indirectly by ROR $\gamma$.

\section{METHODS}

\section{Materials}

Infinity cholesterol (catalog \# TR13421) and Infinity triglyceride (catalog \# TR22421) reagent kits were purchased from Thermo Scientific (Middletown, VA). TRIzol ${ }^{\mathrm{TM}}$ (catalog \#15596018) was purchased from 


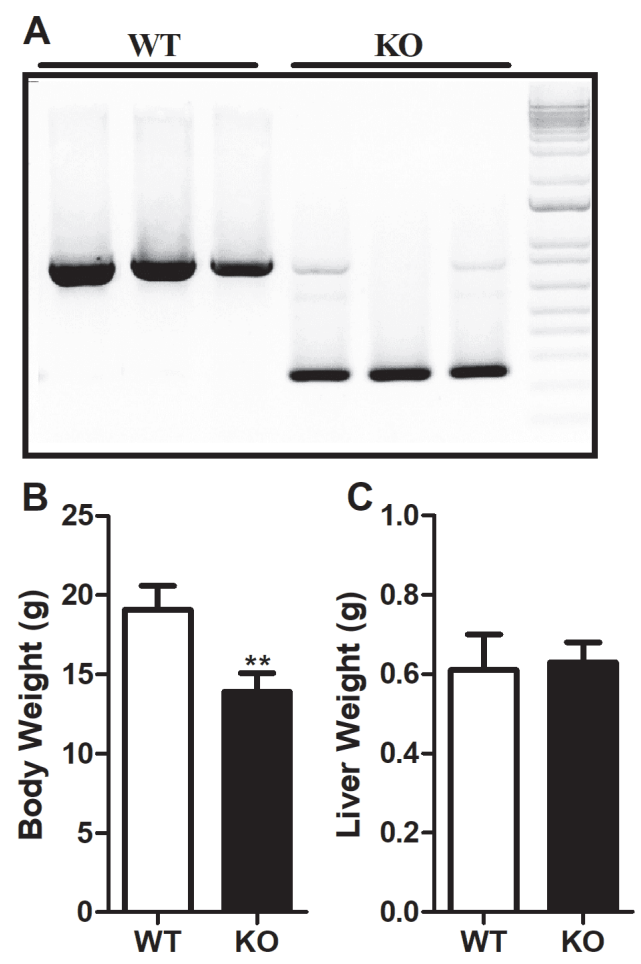

Figure 1. Effect of $R O R \gamma$ gene deletion on body and liver weights in mice. $R O R \gamma$ WT and KO mice $(n=3)$ were identified by genotyping the DNA isolated from tail tissues (A). Total body (B) and liver (C) weights of 8-week old chow diet fed WT and KO mice were measured. Values were plotted as mean \pm SD. $P$ values were calculated using two-tailed Student's $t$ test. ${ }^{\star \star} P<0.01$. WT: wild type; KO: knockout; ROR $\gamma$ : retinoic acid-related orphan receptor $\gamma$

Life Technologies (Carlsbad, CA). Omniscript RT kit (catalog \#205113) was from Qiagen (Germantown, $\mathrm{MD}$ ). $\mathrm{qPCR}^{\mathrm{TM}}$ core kit for SYBR Green I (catalog \#10-SN10-05) was purchased from Eurogentec (San Diego, CA). All other chemicals and solvents were obtained from Fisher Scientific through its local distributor in the Kingdom of Saudi Arabia.

\section{Animals}

Heterozygous ROR $\gamma$ mice were intercrossed to obtain Rory-/- and wildtype (WT) littermate controls as described previously ${ }^{[20]}$. Genotyping was performed by PCR analysis of tail DNA using 5'-TGGTAGTGTCACTATCTGTGTCCC-3' forward and 5'-CTTCAGAACTTATGTCAGGAACTCC-3' reverse primers to generate an 850 -bp WT product and a 250-bp KO product to identify the WT and Rory KO mice [Figure 1A]. For this study, we used eight-week-old chow-diet-fed WT and Rory KO male mice $(n=3)$ on $\mathrm{C} 57 \mathrm{BL} / 6 \mathrm{~J}$ background. All experiments were approved by Institutional Animal Care and Use Committee at King Faisal Specialist Hospital and Research Center.

\section{Body weight and lipid analysis}

Mice in WT and Rory KO groups were fasted overnight for $18 \mathrm{~h}$, weighed using digital balance to measure total body weight and sacrificed to collect blood and livers. Liver weight of each animal was recorded. Blood was used to isolate plasma after centrifugation of the samples for $15 \mathrm{~min}$ at $2500 \mathrm{rpm}$ using a tabletop centrifuge. Plasma and liver tissues were stored at $-80{ }^{\circ} \mathrm{C}$ until further analysis. Hepatic lipids from WT and KO mice were extracted by Bligh and Dyer method ${ }^{[21]}$. Total cholesterol and triglyceride levels in the plasma and liver tissues were measured using commercially available kits from Thermo Scientific as described previously ${ }^{[22]}$. 
Table 1. List of primers used for the quantification of lipid metabolism genes

\begin{tabular}{|c|c|c|}
\hline Gene & Forward primer & Reverse primer \\
\hline \multicolumn{3}{|c|}{ Cholesterol metabolism genes } \\
\hline Hmgr & AGCTTGCCCGAATTGTATGT & TCTGTTGTGAACCATGTGAC \\
\hline Hmgs & TGGCTATAAAGCTGCGGAGG & GGTGAAAGAGCCAAAGGGGA \\
\hline Abcal & GGACATGCACAAGGTCCTGA & CAGAAAATCCTGGAGCTTCA \\
\hline Abcg1 & GCTGTGCGTTTTGTGCTGTT & TGCAGCTCCAATCAGTAGGC \\
\hline Abcg5 & AGGGCCTCACATCAACAGAG & GCTGACGCTGTAGGACACAT \\
\hline Abcg8 & AGTGGTCAGTCCAACACTCT & GAGACCTCCAGGGTATCTTG \\
\hline Acat1 & CCAATGCCAGCACACTGAAC & TCTACGGCAGCATCAGCAAA \\
\hline Acat2 & TGTTGAAAGGTGGGCAGCAA & CAGGTAACATCCCATCCCGT \\
\hline Cyp7a & CAACGGGTTGATTCCATACC & ATTTCCCCATCAGTTTGCAG \\
\hline \multicolumn{3}{|c|}{ Fatty acid metabolism genes } \\
\hline Cd36 & TGCACCACATATCTACCAAA & TTGTAACCCCACAAGAGTTC \\
\hline Fabpl & CAATAGGTCTGCCCGAGGAC & CAGGGTGAACTCATTGCGGA \\
\hline Fas & GGGTTCTAGCCAGCAGAGTC & TCAGCCACTTGAGTGTCCTC \\
\hline Accla & GAGGAAGTTGGCTATCCAGT & CTTGAACCTGTCTGAAGAGG \\
\hline Acc2 & TGTCCCAGGAGGCTGCATTG & TGTGCAGGTCCAGTTTCTTG \\
\hline Scd1 & CCGGAGACCCCTTAGATCGA & TAGCCTGTAAAAGATTTCTG \\
\hline \multicolumn{3}{|c|}{ Triglycerides metabolism genes } \\
\hline Dgat1 & GTTCAGCTCAGACAGTGGTT & TCAGCATCACCACACACCAA \\
\hline Dgat2 & AGCTGCAGGTCATCTCAGTA & CTGCAGGCCACTCCTAGCAC \\
\hline Mgat2 & GAGCAAAGCCCGTGTGTAGA & AAGGTCTGTAACCTGCGCTC \\
\hline Mttp & CACACAACTGGCCTCTCATTAAAT & TGCCCCCATCAAGAAACACT \\
\hline VIdlr & GCCATATGAGAACATGCCGC & AGGACACGGGGATACACTGA \\
\hline Ldlr & TGACTCAGACGAACAAGGCT & ATCTAGGCAATCTCGGTCTC \\
\hline Plin1 & TGCTGCACGTGGAGAGTAAG & AGCAGGGTTGGGCCCTTGTT \\
\hline$L p /$ & TTTGGCTCCAGAGTTTGACC & TGTGTCTTCAGGGGTCCTTA \\
\hline Apoc2 & CATGGGGTCTCGGTTCTTCC & CTTAAGAGGGAGCCCTGCAC \\
\hline \multicolumn{3}{|c|}{ Lipid metabolism transcription factor genes } \\
\hline Srebpla & CCATGGACGAGCTGGCCTTC & AGTTGGCACCTGGGCTGCTG \\
\hline Srebp1c & GGAAGCTGTCGGGGTAGCGT & CATGTCTTCAAATGTGCAAT \\
\hline Srebp2 & GCGTTCTGGAGACCATGGAG & GAGCTACAAAGTTGCTCTGA \\
\hline Ppara & GCGTACGGCAATGGCTTTAT & GAACGGCTTCCTCAGGTTCT \\
\hline Pparg & ATGGTGCCTTCGCTGATGCA & TGGCATCTCTGTGTCAACCA \\
\hline \multicolumn{3}{|c|}{ Housekeeping gene } \\
\hline 18 sRna & GTAACCCGTTGAACCCCATT & CCATCCAATCGGTAGTAGCG \\
\hline
\end{tabular}

\section{Analysis of gene expression by quantitative real time PCR}

TRIzol $^{\mathrm{TM}}$ was used to isolate total RNA from tissues as per the manufacturer's instructions. cDNA was synthesized using Omniscript RT kit from good quality RNA preparations with the purity of RNA having $\mathrm{A}_{260} / \mathrm{A}_{280}$ ratio of more than 1.7. Quantitative PCR in each reaction was carried out in a total volume of $20 \mu \mathrm{L}$, consisting of $5 \mu \mathrm{L}$ of 1:100 diluted first strand cDNA sample and $15 \mu \mathrm{L}$ of SYBR Green I PCR master mix solution. The reaction mixture was incubated for $10 \mathrm{~min}$ at $95{ }^{\circ} \mathrm{C}$ followed by 40 cycles of $15 \mathrm{~s}$ incubations at $95{ }^{\circ} \mathrm{C}$ and $60 \mathrm{~s}$ at $60{ }^{\circ} \mathrm{C}$ in a QuantStudio ${ }^{\mathrm{TM}} 6$ Flex Real-Time PCR (Applied Biosystems). Data were analyzed and presented as arbitrary units that were normalized to the expression of 18 sRNA, according to the manufacturer's instructions. The primers were designed using Primer Express 3.0 (Applied Biosystems) and are shown in Table 1.

\section{Statistical analysis}

Data are presented as mean \pm SD Statistical significance $(P<0.05)$ was determined using Student's $t$ test (GraphPad Prism 5).

\section{RESULTS}

\section{Effect of Rory gene deletion on body and liver weights}

Age matched eight-week-old male Rory KO mice and littermate controls on chow diet were used to assess the effect of Rory gene deletion on total body and liver weights. Rory gene deletion resulted in a significant 

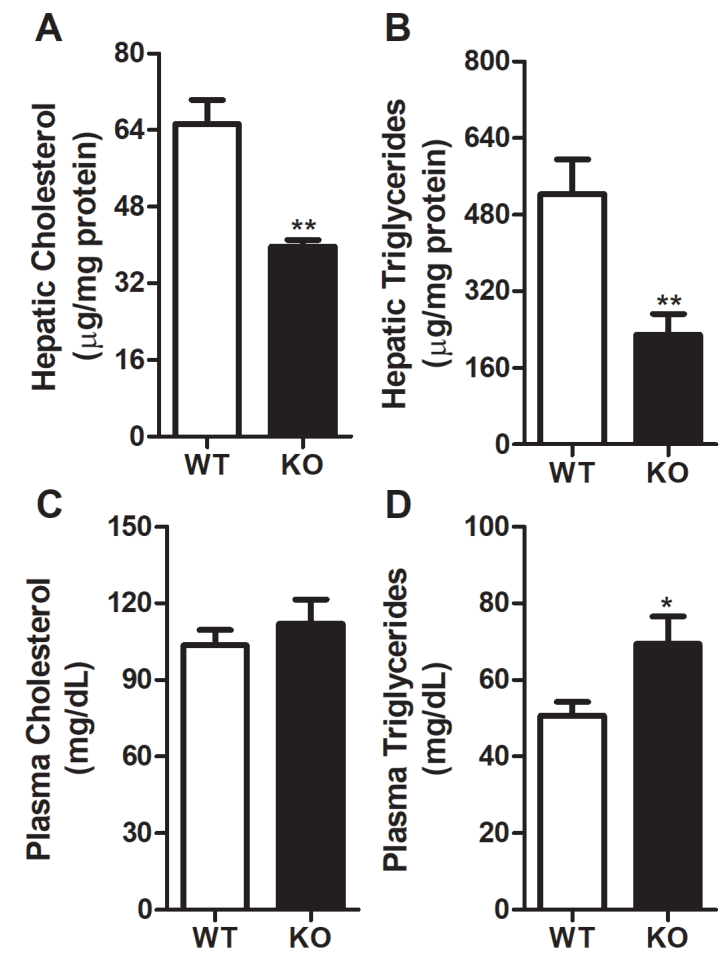

Figure 2. $R O R \gamma$ gene deletion reduces hepatic lipids and increases plasma triglycerides. Lipids from WT and KO mice livers $(n=3)$ were extracted by Bligh and Dyer method to measure cholesterol (A) and triglycerides (B). Total cholesterol (C) and triglycerides (D) were also measured in the plasma of these mice. Values were plotted as mean \pm SD. $P$ values were calculated using two-tailed Student's $t$ test. ${ }^{\star \star} P<$ 0.01. WT: wild type; KO: knockout; ROR $\gamma$ : retinoic acid-related orphan receptor $\gamma$

decrease of $27 \%$ in total body weight compared to WT littermate control mice [Figure 1B]. However, no change was seen in the weight of livers in these mice [Figure $1 \mathrm{C}$ ]. These results suggest that ROR $\gamma$ is important in maintaining the body weight in mice and its deficiency leads to reduced body weight gain.

\section{ROR $\gamma$ KO mice decreases hepatic but not plasma lipids}

Next, we investigated whether change in body weight after Rory gene deletion also affects liver and plasma lipids. Our data indicate that there were significant decreases of $39 \%$ and $56 \%$ in the levels of liver cholesterol [Figure 2A] and triglycerides [Figure 2B], respectively, in Rory KO mice compared to WT littermates. However, we did not see any significant change in plasma cholesterol levels between WT and Rory KO mice [Figure 2C]. Contrary to decreased hepatic lipid levels, we observed an increase of $\sim 37 \%$ in the levels of plasma triglycerides in KO mice compared to WT mice [Figure 2D]. These data suggest that Rory gene deletion affects only liver and not plasma cholesterol. On the other hand, both liver and plasma triglycerides are altered after the ablation of Rory gene.

\section{Ablation of Ror $\gamma$ activity affects expression of genes involved in lipid metabolism}

To gain better understanding of how Rory activity regulates body weight and lipids, we looked at the changes in the expression of lipid metabolism genes after the deletion of Rory gene. We started by analyzing the changes in the genes that regulate cholesterol homeostasis in the liver. Coordinated expression of several genes regulate cellular cholesterol metabolism ${ }^{[2,24]}$. We looked at the expression of cholesterol biosynthesis genes such as hydroxymethylglutaryl-CoA reductase (Hmgr) and hydroxymethylglutarylCoA synthase (Hmgs). The expression of rate-limiting enzyme gene, Hmgr, was not affected by the deletion of Rory in the liver [Figure 3A]. However, we observed a decrease of $63 \%$ in the expression of Hmgs in the livers of Rory KO mice compared to WT littermates [Figure 3B]. Next, we looked at the expression of genes 

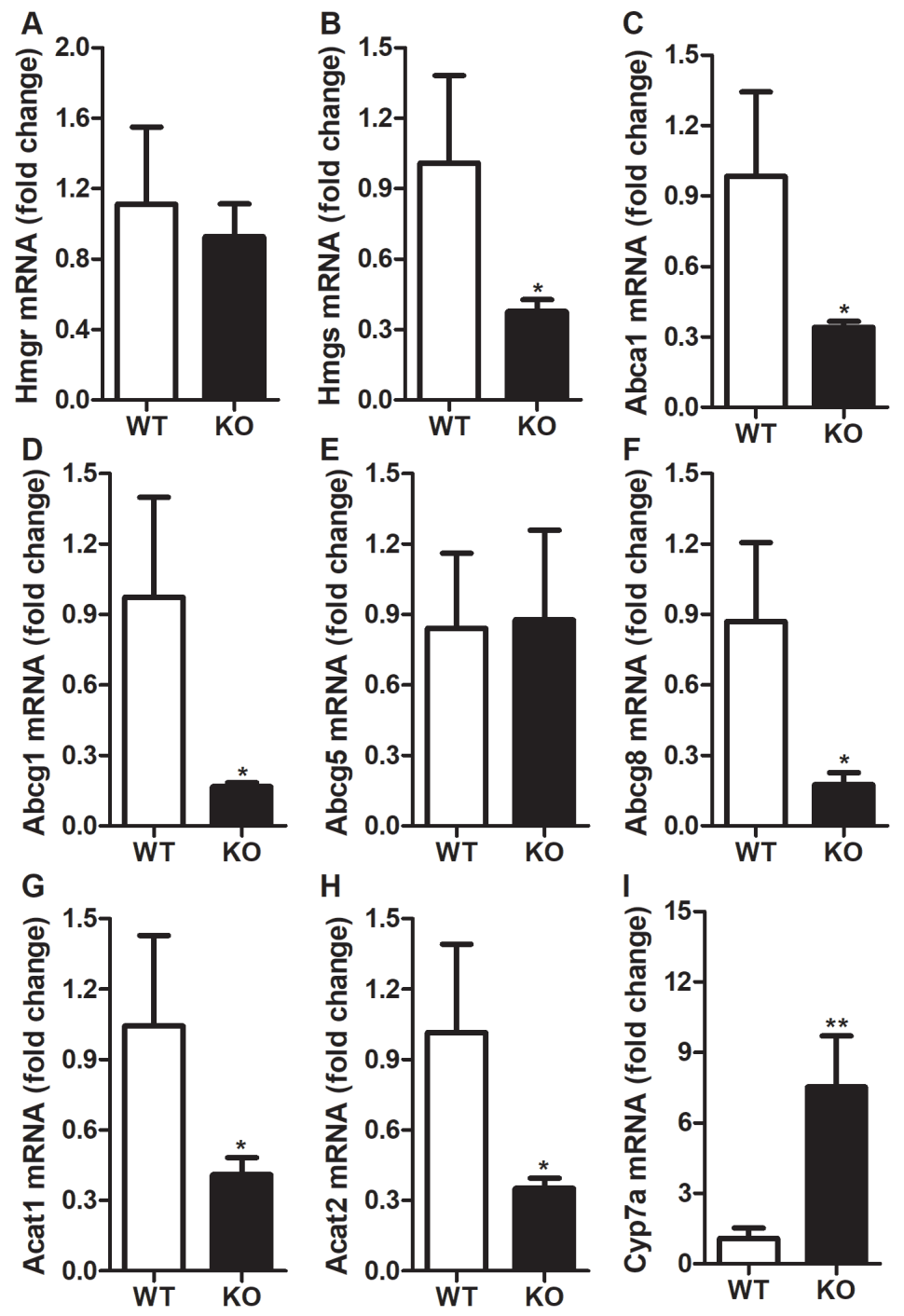

Figure 3. Changes in hepatic expression of cholesterol metabolism genes in $R O R \gamma$ knockout mice. Total mRNA from the livers of WT and $\mathrm{KO}$ mice were used to determine the expression of cholesterol metabolism genes. Relative changes in the mRNA expression were calculated based on the expression of $18 \mathrm{sRNA}$. Values were plotted as mean \pm SD. $P$ values were calculated using two-tailed Student's $t$ test. ${ }^{\star} P<0.05$ and ${ }^{\star \star} P<0.01$. WT: wild type; KO: knockout; ROR $\gamma$ : retinoic acid-related orphan receptor $\gamma ;$ Hmgr: hydroxymethylglutarylCoA reductase; Hmgs: hydroxymethylglutaryl-CoA synthase; Abca1: ATP-binding cassette transporter A1; Abcg1: ATP-binding cassette transporter G1; Abcg5: ATP-binding cassette transporter G5; Abcg8: ATP-binding cassette transporter G8; Acat1: acetyl-CoA acetyltransferase 1; Acat2: acetyl-CoA acetyltransferase 2; Cyp7a: cholesterol 7alpha-hydroxylase

involved in the efflux of cholesterol in the liver. There was a decrease of $65 \%-83 \%$ in the expression of ATPbinding cassette transporter A1 (Abca1), G1 (Abcg1), and G8 (Abcg8) but not G5 (Abcg5) [Figure 3C-F]. Besides the decrease in cholesterol biosynthesis and efflux genes, we also observed a $61 \%-65 \%$ decrease in the expression of acetyl-CoA acetyltransferase 1 (Acat1) and acetyl-CoA acetyltransferase 2 (Acat2) genes that are involved in the esterification of cholesterol for storage and lipoprotein secretion [Figure $3 \mathrm{G}$ and $\mathrm{H}$ ]. On the other hand, expression of cholesterol 7alpha-hydroxylase (Сур7a) involved in the biosynthesis of bile acids was increased by $\sim 6$ folds in the livers of Rory KO mice [Figure 3I]. Taken together, these results demonstrate that ablation of Rory activity results in decreased expression of genes involved in cholesterol biosynthesis, efflux, and storage but increased expression of genes involved in bile acid biosynthesis.

We then looked at the changes in the expression of fatty acid metabolism genes. Uptake of long-chain fatty acids by the liver is dependent on fatty acid transporters such as cluster of differentiation $36(\mathrm{Cd} 36)$. 

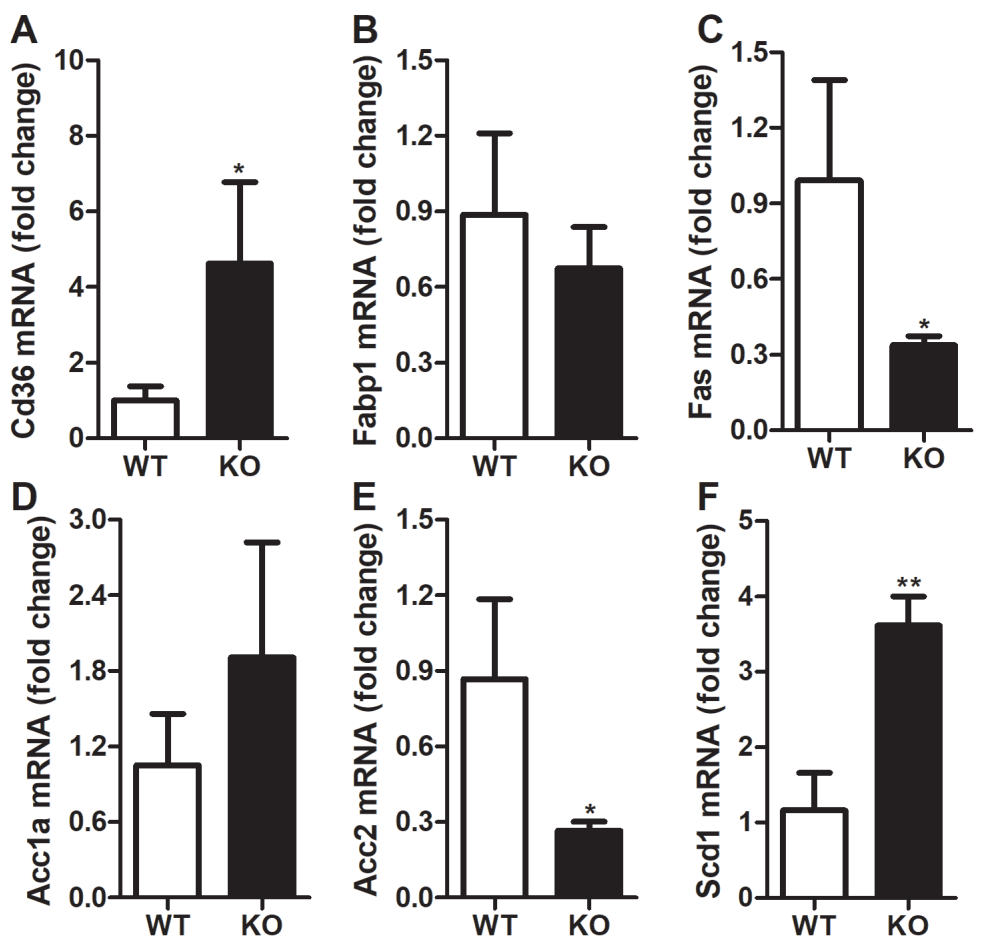

Figure 4. Changes in hepatic expression of fatty acid metabolism genes in ROR $\gamma$ knockout mice. Total mRNA from the livers of WT and $\mathrm{KO}$ mice were used to determine the expression of fatty acid metabolism genes. Relative changes in the mRNA expression were calculated based on the expression of $18 \mathrm{sRNA}$. Values were plotted as mean \pm SD. $P$ values were calculated using two-tailed Student's $t$ test. ${ }^{\star} P<0.05$ and ${ }^{\star \star} P<0.01$. WT: wild type; KO: knockout; ROR $\gamma$ : retinoic acid-related orphan receptor $\gamma ; C d 36$ : cluster of differentiation 36; Fabp1: fatty acid binding protein 1; Fas: fatty acid synthase; Acc1a: acetyl-CoA carboxylase 1a; Acc2: acetyl-CoA carboxylase 2; Scd1: stearoyl-coenzyme A desaturase-1

There was a $\sim 4.5$-fold increase in the expression of $C d 36$ in the livers of $\mathrm{KO}$ mice compared to WT mice [Figure $4 \mathrm{~A}$ ]. However, we did not see any change in the expression of fatty acid binding protein 1 (Fabp1) in the livers of Rory KO mice [Figure 4B]. Next, we looked at the expression of fatty acid synthesis genes and found that deletion of Rory decreased the expression of fatty acid synthase (Fas) and acetylCoA carboxylase $2(A c c 2)$ by $66 \%$ [Figure $4 \mathrm{C}$ ] and 70\% [Figure $4 \mathrm{E}$ ], respectively, but had no significant effect on the expression of acetyl-CoA carboxylase 1a (Acc1a) [Figure 4D]. Interestingly, expression of stearoyl-coenzyme A desaturase-1 $(S c d 1)$, which catalyzes the conversion of saturated fatty acids to monounsaturated fatty acids, was increased by $\sim 3$ folds in the livers of Rory KO mice [Figure 4F]. These results suggest that deletion of Rory increases the expression of genes involved in fatty acids uptake and desaturation but decreases the expression of genes involved in their biosynthesis.

We further investigated the effect of Rory gene deletion on the expression of genes involved in triglycerides metabolism. There was a significant reduction of $77 \%$ in the expression of diacylglycerol O-acyltransferase 2 (Dgat2) gene [Figure 5B] in contrast to a small but not significant increase in the expression of Dgat1 gene [Figure 5A], and no change in the expression of acyl CoA: monoacylglycerol acyltransferase 2 (Mgat2) gene [Figure $5 \mathrm{C}$ ] in the livers of Rory KO mice as compared to WT mice. Dgat2 is mainly expressed and involved in the synthesis of bulk of triglycerides in mouse liver in contrast to Dgat1 and Mgat2, which are highly expressed in the small intestine ${ }^{[25,26]}$. Our data also show that deletion of Rory gene did not affect the expression of microsomal triglyceride transfer protein (Mttp) gene, a key protein involved in the assembly and secretion of lipid rich lipoproteins ${ }^{[27,28]}$ [Figure 5D]. Very low-density lipoprotein receptor (Vldlr) and low-density lipoprotein receptor $(L d l r)$ are important proteins that regulate the uptake of lipid rich lipoproteins from the circulation. There was no significant difference in the expression of Vldlr between the 

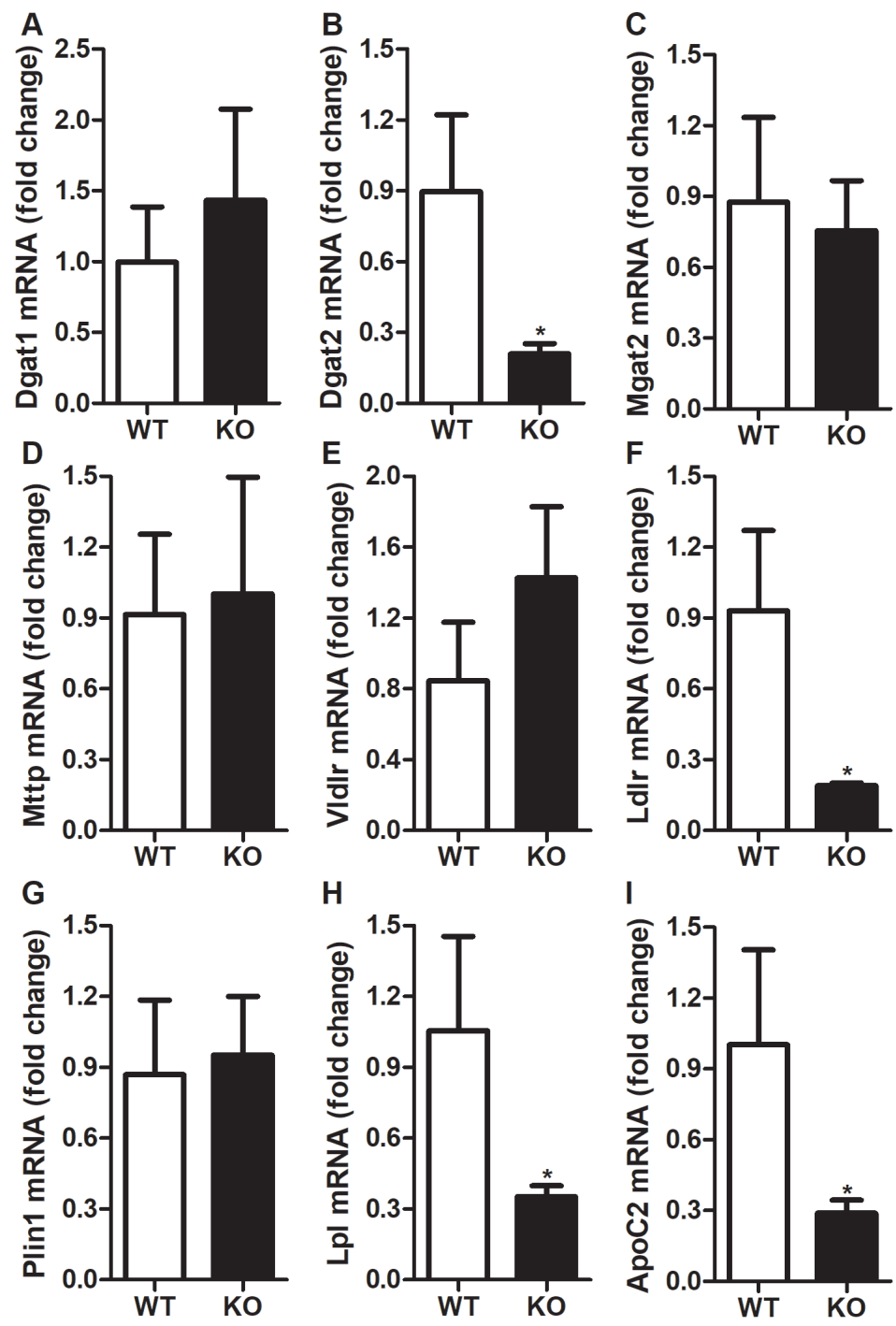

Figure 5. Changes in hepatic expression of triglycerides metabolism genes in $R O R \gamma$ knockout mice. Total mRNA from the livers of WT and $\mathrm{KO}$ mice were used to determine the expression of triglycerides metabolism genes. Relative changes in the mRNA expression were calculated based on the expression of 18 sRNA. Values were plotted as mean \pm SD. $P$ values were calculated using two-tailed Student's $t$ test. ${ }^{\star} P<0.05$. WT: wild type; KO: knockout; ROR $\gamma$ : retinoic acid-related orphan receptor $\gamma$; Dgat1: diacylglycerol O-acyltransferase 1; Dgat2: diacylglycerol O-acyltransferase 2; Mgat2: acyl CoA:monoacylglycerol acyltransferase 2; Mttp: microsomal triglyceride transfer protein; VIdlr: very low-density lipoprotein receptor; Ldlr: low-density lipoprotein receptor; Plin1: perilipin 1; Lpl: lipoprotein lipase; Apoc2: apolipoprotein C2

Rory KO and WT mice [Figure 5E] but we observed a 79\% decrease in Ldlr gene expression in the livers of KO mice compared to WT mice [Figure 5F]. Furthermore, there was no difference in the expression of lipid droplet protein, perilipin 1 (Plin1), in the livers of Rory KO and WT mice [Figure 5G]. To understand the reasons for the increase in plasma triglycerides, we looked at the expression of lipoprotein lipase ( $L p l)$ and apolipoprotein $\mathrm{C} 2(A p o C 2)$, which are involved in the lipolysis of lipids in the circulation. Expression of both these genes was reduced by $67 \%-71 \%$ in Rory KO mice [Figure $5 \mathrm{H}$, I]. These results suggest that expression of genes involved in triglycerides synthesis, uptake, and lipolysis is decreased in Rory KO mice leading to decreased triglycerides accumulation in the liver and increased triglycerides in the plasma.

To address whether alterations in cellular lipids is due to the changes in the expression of nuclear transcription factors, we looked at the expression of these genes in the liver. Our data indicate that the deletion of Rory reduced Srebp2 gene expression by $80 \%$ in the livers [Figure 6A]. These data are consistent 
with the lower hepatic cholesterol levels [Figure 2A] and decreased expression of downstream genes involved in cholesterol biosynthesis pathway [Figure 3]. Our results show that ablation of Rory activity in the liver leads to a $68 \%$ decrease in the expression of Srebp1c gene [Figure 6B], which is consistent with the reduced hepatic triglycerides [Figure 2B] and lower expression of the lipid synthesis genes [Figures 4 and 5]. Furthermore, we also noticed that expression of Srebp 1 a gene was significantly reduced by $62 \%$ in the livers of Rory KO mice compared to WT littermates [Figure 6C]. Next, we looked at the expression of Ppara and Pparg genes in the livers of Rory KO and WT mice. There was an around five-fold increase in the expression of Ppara gene, which is mainly involved in fatty acid oxidation [Figure 6D]. However, we observed a decrease of $74 \%$ in the expression of Pparg gene [Figure 6E]. These data suggest that expression of transcription factors that regulate lipid metabolism is modulated by $\mathrm{ROR} \gamma$.

\section{DISCUSSION}

Our results identify a critical role of ROR $\gamma$ in lipid metabolism. Here, we show that ablation of Rory gene decreases the body weight and reduces the accumulation of lipids in the liver. Interestingly, we did not see any significant change in the liver weight between the WT and Rory KO mice [Figure 1C]; however, we noticed a visible decrease in the content of abdominal fat mass in Rory KO mice (data not shown). This decrease in body weight and fat mass may be due to either reduced food intake or increased energy expenditure. It is known that activation of PPAR $\gamma$ by agonists promote weight gain and fat accumulation mainly due to increased adipocyte differentiation and lipid storage ${ }^{[29,30]}$. Our data indicate that Rory gene deletion results in lower expression of Pparg [Figure 6E], which may explain the decrease in body weight and fat mass. This finding is consistent with the repression of Rory activity by inverse agonist that lead to a reduction in fat mass and body weight in obese diabetic mice ${ }^{[19]}$.

A decrease in body weight and fat mass in Rory $\mathrm{KO}$ mice suggests that these mice do not store enough lipids in the adipocytes. Inability of adipocytes to store the excess fatty acids coming from the diet suggest that these fatty acids may be taken up by the liver for either oxidation or synthesis of lipids for storage in hepatic or extrahepatic tissues such as muscles. A significant increase in the hepatic expression of $C d 36$ gene responsible for the uptake of long-chain fatty acids suggests that the livers from Rory KO mice may be taking up more fatty acids from the circulation [Figure $4 \mathrm{~A}]$. Takeda et al. ${ }^{[17]}$ also showed that expression of $\mathrm{Cd} 36$ gene was upregulated at all the times during diurnal oscillations in the livers of Rory KO mice. Since both cholesterol and triglycerides levels were reduced in the livers of Ror $\mathrm{KO}$ mice [Figure 2A and B], we speculate that most of the fatty acids taken up by these mice are not utilized for lipid biosynthesis and storage. This was further supported by our observation that the expression of genes involved in lipid biosynthesis was lower in the livers from Rory $\mathrm{KO}$ mice [Figure 5B]. It is interesting to note that there was a reduction in the expression of Acc2 in the livers of Rory KO mice. This enzyme is involved in the synthesis of malonyl-coA that plays an important role in regulating the oxidation of fatty acids in the mitochondria by inhibiting the carnitine/palmitoyl shuttle system ${ }^{[31]}$ in contrast to malonyl-coA that is generated by the ACC1 and utilized by Fas for the synthesis of fatty acids in the cytosol ${ }^{[32]}$. Therefore, lower levels of Acc2 gene expression suggests that the oxidation of fatty acids may be enhanced in the livers of Rory KO mice, which is consistent with the inhibition of Rory activity by inverse agonist that leads to enhanced fatty acid oxidation $^{[19]}$. Hence, besides reduced lipid biosynthesis, increased fatty acid oxidation may also contribute to reduced accumulation of triglycerides in the livers of Rory KO mice.

Contrary to lower hepatic lipids, we observed an increase in the levels of triglycerides in the plasma of Rory KO mice. On the other hand, Takeda et al. ${ }^{[17]}$ reported a decrease in the levels of plasma lipids in Rory KO mice. The discrepancy in results may be due to the feeding of high fat diet for six weeks to these mice, which may affect the metabolism of lipids in the plasma. It is likely that Rory gene ablation affects the uptake or secretion of these lipids from the liver. We did not observe any changes in the expression of $M t t p$, a gene responsible for the secretion of lipid rich lipoproteins, suggesting that secretion of lipids 

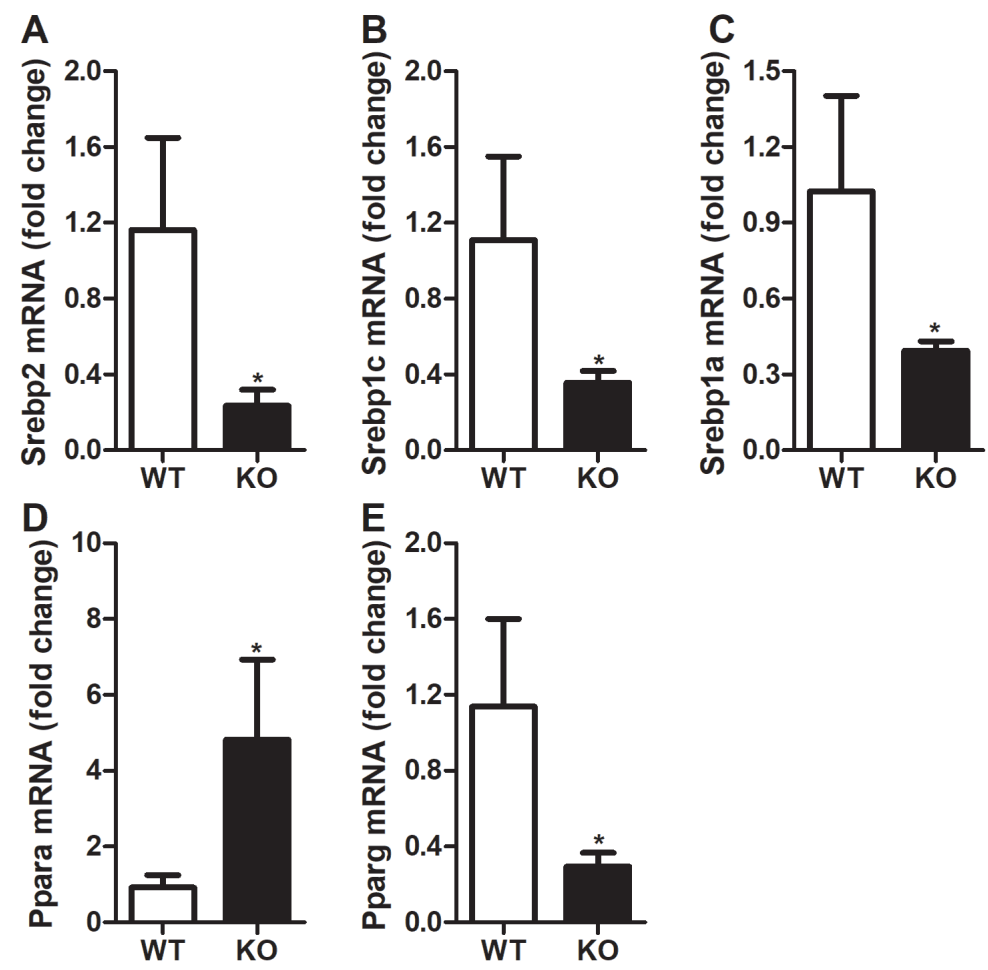

Figure 6. Changes in hepatic expression of lipid metabolism transcription factor genes in RORy knockout mice. Total mRNA from the livers of WT and KO mice were used to determine the expression of transcription factor genes. Relative changes in the mRNA expression were calculated based on the expression of 18 SRNA. Values were plotted as mean \pm SD. $P$ values were calculated using two-tailed Student's $t$ test. ${ }^{\star} P<0.05$. WT: wild type; KO: knockout; ROR $\gamma$ : retinoic acid-related orphan receptor $\gamma ;$ Srebp2: sterol regulatory-element binding protein 2; Srebp1c: sterol regulatory-element binding protein 1c; Srebp1a: sterol regulatory-element binding protein 1a; Ppara: peroxisome proliferator-activated receptor $\alpha$; Ppar $\gamma$ : peroxisome proliferator-activated receptor $\gamma$

by the liver may not be affected by Rory gene deletion. However, our data indicate that Rory gene deletion decrease the levels of $L d l r$ gene expression implicating that uptake of lipid rich lipoproteins may be reduced in the $\mathrm{KO}$ mice leading to increase in the levels of plasma triglycerides. It is possible that the increase in plasma triglycerides may also be due to reduced activity of lipoprotein lipase. In fact, our data show that expression of $\mathrm{Lpl}$ and $\mathrm{ApoC} 2$ genes were reduced in the livers of Rory $\mathrm{KO}$ mice. ApoC2 acts as an activator of lipoprotein lipase ${ }^{[33]}$ and its reduction in the circulation may lead to reduced lipolysis, thereby causing increased plasma triglycerides.

ROR $\gamma$ functions as a transcriptional mediator that has been shown to regulate the rhythmic expression of certain metabolic genes downstream of clock machinery ${ }^{[17,18]}$. Regulation of these genes is complex and may involve other nuclear receptors or transcriptional factors downstream of Rory to modulate lipid metabolism directly or indirectly ${ }^{[17]}$. There is also a possibility that changes in these transcriptional factors may be secondary to the changes in the homeostasis of lipids in the cells ${ }^{[34]}$. Expression of several lipid metabolism genes is regulated by various SREBPs that sense the changes in lipid levels in the cells. Stimulation of SREBP-2 regulates cholesterol homeostasis by activating the transcription of genes involved cholesterol metabolism ${ }^{[35]}$. On the other hand, stimulation of SREBP-1c increases lipogenesis by increasing the expression of fatty acid metabolism genes ${ }^{[36]}$. SREBP-1a has been shown to be an important regulator of both cholesterol and lipid synthesis genes ${ }^{[37]}$. Our results suggest that deletion of Rory decreases the expression of Srebp1a, Srebp1c, and Srebp2 in the liver [Figure 6]. Changes in the expression of these transcription factors decreased the overall biosynthesis of cholesterol, fatty acids, and triglycerides in the liver cells. Our data also indicate that deletion of Rory may increase the oxidation of fatty acids due to increased expression of Ppara in the liver. 
We observed that expression of genes involved in biosynthesis, efflux, and esterification of cholesterol was down-regulated in Rory KO mice [Figure 3]. This may be the reason for less cholesterol levels in the livers of these mice [Figure 2A]. Interestingly, we noticed a significant increase in the expression of Cyp7a gene, which is responsible for the biosynthesis of bile acids in the liver. Although we do not know the exact mechanism of how hepatic bile acid signaling was impaired in Rory KO, we speculate that a larger portion of the hepatic cholesterol pool may be directed towards bile acid biosynthesis in the absence of ROR $\gamma$. Takeda et al. ${ }^{[17]}$ also observed an increase in the expression of Cyp $7 a$ gene in Rory KO mice fed a chow diet. However, when these mice were fed a high fat diet for eight weeks, the levels of their liver, serum, and fecal bile acid pools were reduced significantly. It is possible that the high fat diet is repressing the expression of some other genes involved in the bile acid biosynthesis pathway.

In this study, we demonstrated that ROR $\gamma$ plays a significant role in the regulation of the expression of lipid metabolism genes that are involved in lipid biosynthesis, storage, and fatty acid oxidation. Our data also suggest that a cross-talk may occur between various transcription factors to maintain overall lipid homeostasis in the body. Previously, it has been shown that hepatic circadian clock plays a critical role in regulating major aspects of lipid metabolism through ROR $\gamma$ activity ${ }^{[17]}$. In a different experimental setting, we expanded the investigation to look at the changes in several lipid metabolism genes that were not studied previously. We also looked at the expression of different transcription factors to study the cross-talk between ROR $\gamma$ and these transcription factors to regulate lipid homeostasis. Most of the findings in this current study are consistent and added substantive findings to the previously published reports ${ }^{[17,19]}$.

In summary, we show here that ablation of ROR $\gamma$ activity reduce lipid biosynthesis but enhance fatty acid oxidation by modulating the expression of several lipid metabolism genes. However, this study has some limitations because global ROR $\gamma$-deficient mice are born healthy and fertile but die within the first four months after birth due to high incidence of T-cell lymphomas in the thymus ${ }^{[15]}$. Therefore, further studies are warranted to show the effect of ROR $\gamma$ deficiency on lipid metabolism in liver-specific KO mice to avoid any complications or confounding effects due to T-cell lymphomas. We will extend the studies of Takeda et al. ${ }^{[17]}$ to investigate role of ROR $\gamma$ on lipid metabolism genes in chow-fed mice that were not studied previously. We will also look at the expression of different transcription factors to study the cross-talk between these factors and RORg to regulate lipid homeostasis. Furthermore, these mice may also be a good model to study the effect of liver-specific Rory gene deletion on diet-induced obesity. In conclusion, it may be beneficial to target ROR $\gamma$ activity for the management of obesity and related lipid metabolic disorders.

\section{DECLARATIONS}

\section{Authors' contributions}

Conducted experiments: Jahangir Z, Veluru D, Otaibi AA, Mubarak SA, Subie BA, Alghanem AF

Contributed to the conception and design of the study: Iqbal J, Hawwari A, Bakillah A, Qarni AA

Performed data analysis and interpretation: Iqbal J, Bakillah A

Wrote the paper: Iqbal J, Bakillah A, Hawwari A

\section{Availability of data and materials}

All data and materials and mice are publicly available.

\section{Financial support and sponsorship}

This research was supported by National Science, Technology and Innovation Plan's strategies technologies program in Saudi Arabia (KACST: 10-BIO950-20) and an internal fund (RAC \# 2083003) by KFSH\&RC to A.H. This work was also partially supported by the intramural grants RA16/024/A and RA17/013/A to Iqbal J and RA17/014/A to Alghanem AF from KAIMRC. Jahangir Z worked on this study as a high school summer trainee at KAIMRC. 


\section{Conflicts of interest}

All authors declare that there are no conflicts of interest.

\section{Ethical approval and consent to participate}

All experiments were approved by Institutional Animal Care and Use Committee at King Faisal Specialist Hospital and Research Center.

\section{Consent for publication}

Not applicable.

\section{Copyright}

(c) The Author(s) 2019 .

\section{REFERENCES}

1. Rutkowski JM, Stern JH, Scherer PE. The cell biology of fat expansion. J Cell Biol 2015;208:501-12.

2. Van den BG. The role of the liver in metabolic homeostasis: implications for inborn errors of metabolism. J Inherit Metab Dis 1991; 14:407-20.

3. Karagianni P, Talianidis I. Transcription factor networks regulating hepatic fatty acid metabolism. Biochim Biophys Acta 2015;1851:28.

4. Guo Y, Jolly RA, Halstead BW, Baker TK, Stutz JP, et al. Underlying mechanisms of pharmacology and toxicity of a novel PPAR agonist revealed using rodent and canine hepatocytes. Toxicol Sci 2007;96:294-309.

5. Schoonjans K, Watanabe M, Suzuki H, Mahfoudi A, Krey G, et al. Induction of the acyl-coenzyme A synthetase gene by fibrates and fatty acids is mediated by a peroxisome proliferator response element in the C promoter. J Biol Chem 199518;270:19269-76.

6. Jump DB. Fatty acid regulation of gene transcription. Crit Rev Clin Lab Sci 2004;41:41-78.

7. Semple RK, Chatterjee VK, O'Rahilly S. PPAR gamma and human metabolic disease. J Clin Invest 2006;116:581-9.

8. Lee YJ, Ko EH, Kim JE, Kim E, Lee H, et al. Nuclear receptor PPARgamma-regulated monoacylglycerol O-acyltransferase 1 (MGAT1) expression is responsible for the lipid accumulation in diet-induced hepatic steatosis. Proc Natl Acad Sci U S A 2012;109:13656-61.

9. Wong RH, Sul HS. Insulin signaling in fatty acid and fat synthesis: a transcriptional perspective. Curr Opin Pharmacol 2010;10:684-91.

10. Horton JD, Goldstein JL, Brown MS. SREBPs: activators of the complete program of cholesterol and fatty acid synthesis in the liver. J Clin Invest $2002 ; 109: 1125-31$.

11. Horton JD, Shah NA, Warrington JA, Anderson NN, Park SW, et al. Combined analysis of oligonucleotide microarray data from transgenic and knockout mice identifies direct SREBP target genes. Proc Natl Acad Sci U S A 200314;100:12027-32.

12. Chawla A, Boisvert WA, Lee CH, Laffitte BA, Barak Y, et al. A PPAR gamma-LXR-ABCA1 pathway in macrophages is involved in cholesterol efflux and atherogenesis. Mol Cell 2001;7:161-71.

13. Solt LA, Burris TP. Action of RORs and their ligands in (patho) physiology. Trends Endocrinol Metab 2012;23:619-27.

14. Medvedev A, Yan ZH, Hirose T, Giguere V, Jetten AM. Cloning of a cDNA encoding the murine orphan receptor RZR/ROR gamma and characterization of its response element. Gene 1996;181:199-206.

15. Ueda E, Kurebayashi S, Sakaue M, Backlund M, Koller B, et al. High incidence of T-cell lymphomas in mice deficient in the retinoidrelated orphan receptor RORgamma. Cancer Res 2002;62:901-9.

16. Jetten AM. Retinoid-related orphan receptors (RORs): critical roles in development, immunity, circadian rhythm, and cellular metabolism. Nucl Recept Signal 2009; 7:e003.

17. Takeda Y, Kang HS, Lih FB, Jiang H, Blaner WS, et al. Retinoid acid-related orphan receptor gamma, RORgamma, participates in diurnal transcriptional regulation of lipid metabolic genes. Nucleic Acids Res 2014;42:10448-59.

18. Jetten AM, Kang HS, Takeda Y. Retinoic acid-related orphan receptors alpha and gamma: key regulators of lipid/glucose metabolism, inflammation, and insulin sensitivity. Front Endocrinol (Lausanne) 2013;4:1.

19. Chang MR, He Y, Khan TM, Kuruvilla DS, Garcia-Ordonez R, et al. Antiobesity effect of a small molecule repressor of RORgamma. Mol Pharmacol 2015;88:48-56.

20. Kurebayashi S, Ueda E, Sakaue M, Patel DD, Medvedev A, et al. Retinoid-related orphan receptor gamma (RORgamma) is essential for lymphoid organogenesis and controls apoptosis during thymopoiesis. Proc Natl Acad Sci U S A 2000;97:10132-7.

21. Bligh EG, Dyer WJ. A rapid method of total lipid extraction and purification. Can J Biochem Physiol 1959;37:911-7.

22. Iqbal J, Rudel LL, Hussain MM. Microsomal triglyceride transfer protein enhances cellular cholesteryl esterification by relieving product inhibition. J Biol Chem 2008;283:19967-80.

23. Rotllan N, Fernandez-Hernando C. MicroRNA Regulation of Cholesterol Metabolism. Cholesterol 2012;2012:847849.

24. Nes WD. Biosynthesis of cholesterol and other sterols. Chem Rev 2011;111:6423-51.

25. Yen CL, Farese RV Jr. MGAT2, a monoacylglycerol acyltransferase expressed in the small intestine. J Biol Chem 2003;278:18532-7.

26. McLaren DG, Han S, Murphy BA, Wilsie L, Stout SJ, et al. DGAT2 inhibition alters aspects of triglyceride metabolism in rodents but 
not in non-human primates. Cell Metab 2018;27:1236-48.

27. Hussain MM, Shi J, Dreizen P. Microsomal triglyceride transfer protein and its role in apoB-lipoprotein assembly. J Lipid Res 2003;44:22-32.

28. Hussain MM, Iqbal J, Anwar K, Rava P, Dai K. Microsomal triglyceride transfer protein: a multifunctional protein. Front Biosci 2003;8:s500-6.

29. Kadowaki T, Hara K, Kubota N, Tobe K, Terauchi Y, et al. The role of PPARgamma in high-fat diet-induced obesity and insulin resistance. J Diabetes Complications 2002;16:41-5.

30. Rosen ED, Sarraf P, Troy AE, Bradwin G, Moore K, et al. PPAR gamma is required for the differentiation of adipose tissue in vivo and in vitro. Mol Cell 1999;4:611-7.

31. McGarry JD, Mannaerts GP, Foster DW. A possible role for malonyl-CoA in the regulation of hepatic fatty acid oxidation and ketogenesis. J Clin Invest 1977;60:265-70.

32. Ruderman NB, Saha AK, Kraegen EW. Minireview: malonyl CoA, AMP-activated protein kinase, and adiposity. Endocrinology 2003;144:5166-71.

33. Fojo SS, Brewer HB. Hypertriglyceridaemia due to genetic defects in lipoprotein lipase and apolipoprotein C-II. J Intern Med 1992;231:669-77.

34. Jump DB, Tripathy S, Depner CM. Fatty acid-regulated transcription factors in the liver. Annu Rev Nutr 2013;33:249-69.

35. Brown MS, Goldstein JL. The SREBP pathway: regulation of cholesterol metabolism by proteolysis of a membrane-bound transcription factor. Cell 1997;89:331-40.

36. Stoeckman AK, Towle HC. The role of SREBP-1c in nutritional regulation of lipogenic enzyme gene expression. J Biol Chem 2002;277:27029-35.

37. Shimano H, Horton JD, Hammer RE, Shimomura I, Brown MS, et al. Overproduction of cholesterol and fatty acids causes massive liver enlargement in transgenic mice expressing truncated SREBP-1a. J Clin Invest 1996;98:1575-84. 